This report was prepared as an account of work sponsored by an agency of the United States Government. Neither the United States Government nor any agency thereof, nor any of their employees, makes any warranty, express or implied, or assumes any legal liability or responsibility for the accuracy, completeness, or usefulness of any information, apparatus, product, or process disclosed, or represents that its use would not infringe privately owned rights. Reference herein to any specific commercial product, process, or service by trade name, trademark, manufacturer, or otherwise does not necessarily constitute or inply its enderemmendation, or favoring by the United States Government or any agency thereof. The views and opinions of authors expressed herein do not necessarily state or reflect those of the United States Government or any agency thereof.

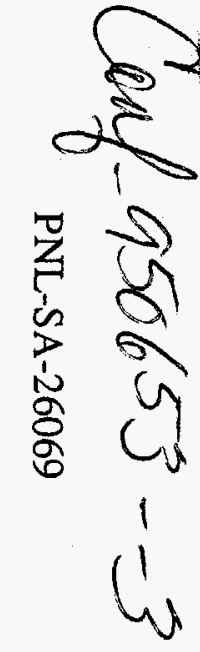




\section{DISCLAIMER}

Portions of this document may be illegible in electronic image products. Images are produced from the best available original document. 


\title{
ADAPTIVE LIFE SIMULATOR: A NOVEL APPROACH TO MODELING THE CARDIOVASCULAR SYSTEM
}

\author{
Lars J. Kangas, Paul E. Keller, Sherif Hashem, Richard T. Kouzes, Paul A. Allen* \\ Pacific Northwest Laboratory ${ }^{\dagger}$ \\ P.O. Box 999, K1-87 \\ Richland, WA 99352 \\ Fax: (509) 375-6631 \\ lj_kangas@pnl.gov and pe_kellerepnl.gov \\ *Life Link \\ 949 Stevens Drive \\ Richland, WA 99352
}

\begin{abstract}
In this paper, an adaptive life simulator (ALS) is introduced. The ALS models a subset of the dynamics of the cardiovascular behavior of an individual by using a recurrent artificial neural network. These models are developed for use in applications that require simulations of cardiovascular systems, such as medical mannequins, and in medical diagnostic systems.

This approach is unique in that each cardiovascular model is developed from physiological measurements of an individual. Any differences between the modeled variables and the actual variables of an individual can subsequently be used for diagnosis. This approach also exploits sensor fusion applied to biomedical sensors. Sensor fusion optimizes the utilization of the sensors. The advantage of sensor fusion has been demonstrated in applications including control and diagnostics of mechanical and chemical processes.
\end{abstract}

\section{Introduction}

The ALS models an individual's cardiovascular system through the relationship between physiological variables at different physical activity levels. The physiological variables include heart rate, systolic and diastolic blood pressures, and breathing rate. Because the model is adapted to an individual, it becomes a model of the physical condition of that individual's cardiovascular system. If a model is developed for a healthy individual, then that model can be compared to a later model of that individual or directly to the individual. Any differences between these models can be exploited to evaluate and diagnose medical conditions that affect the cardiovascular system of that individual.
The ALS used in clinical exercise testing (e.g., graded exercise tests) will increase the sensitivity of correctly diagnosing medical conditions: myocardial ischemia, peripheral vascular disease, exercise-induced asthma, vasoregulatory asthenia, and psychogenic dyspnea. The ALS will also increase the sensitivity of detecting other conditions: chronic bronchitis; pulmonary emphysema; pulmonary infiltration, alveolitis, and fibrosis; pulmonary thromboelism and hypertension; congenital cardiac abnormalities; cardiac valvular obstruction or incompetence. These conditions cannot be uniquely diagnosed in an exercise test alone [1], [2], [3].

A model developed by the ALS can be incorporated into an automatic, continuous diagnostic system carried on a person. Physiological variables, received from noninvasive biomedical sensors, can be compared with the modeled variables in real-time. This real-time diagnosis of an individual's general health increases the possibility of early detection of undesired medical conditions. It can reduce the response time of medical help for people working in hazardous and dangerous environments (e.g., soldiers and law enforcement officers). A real-time diagnostic system also enables continuous monitoring of people with medical conditions in nursing homes and in home-care situations. Reduction of the response time for medical help is critical in minimizing medical complications and the loss of life.

Initially, we expect that employees working in hazardous environments would be monitored for early diagnoses of degradation in health. The working environment and other causes may contribute to a degradation in health that makes an employee unsuitable for certain work. For example, the described system could aid fire districts in determining the health effects from smoke inhalation on individual firemen. The system would determine whether firemen have recovered sufficiently from the last inhalations of smoke and whether they should be allowed

\footnotetext{
This work was supported by the Laboratory Directed Research and Development Program at Pacific Northwest Laboratory (PNL). PNL is a multiprogram national laboratory operated by Battelle Memorial Institute for the U.S. Department of Energy under Contract DE-ACO6-76RLO'1830.
} 
to enter smoke filled environments again. The system will automate these types of testing procedures for workers in hazardous environments.

Other medical conditions applicable to a real-time diagnostic system include detection of hypertension and fatigue. In an individual, these conditions are detected at severity levels in which they change the regular relationships among the modeled physiological variables of the individual.

The ALS is developed using an adaptive technology known as artificial neural networks (ANN). ANNs have been applied to modeling complex process dynamics for the manufacturing and chemical industries. We hypothesize that cardiovascular systems exhibit similar dynamics and can be modeled with ANNs.

\section{Artificial Neural Networks}

An ANN is an algorithmic system implemented in either software or hardware. The concept of ANNs was inspired by the way the biological brain processes information. ANNs, like people, learn by example. Learning in the biological brain occurs in a network of neurons that are interconnected by axons. A point of contact (actually most often a narrow gap) between an axon from one neuron to another is called a synapse. Learning is a matter of adjusting the electrochemical connectivity across these synapses.

An ANN is a network of neurons or processing elements (PE) and weighted connections. The connections correspond to axons and the weights to synapses in the biological brain. A PE performs two functions. It sums the inputs from several incoming connections and then applies a transfer function to the sum. The resulting value is propagated through outgoing connections to other PEs. Typically, these PEs are arranged in layers; with the input layer receiving inputs from the real-world and each succeeding layer receiving weighted outputs from the preceding layer as its input. Hence the creation of a feed forward ANN in which each input is fed forward to its succeeding layer. The first and last layers in this ANN configuration are typically referred to as input and output layers. (Input-layer PEs are not true PEs in that they do not perform a computation on the input.) Any layers between the input and output layers (usually $0-2$ in number) are called hidden layers because they do not have contact with any real-world input or output data.

In addition to simple feedforward ANNs, some feedforward ANNs are recurrent ANNs. These have feedback connections that move intermediately processed data or output data back to previous layers. These feedback connections allow the ANNs to capture temporal information in data and, thus, model dynamic systems.

Back propagation is one of several possible learning rules to adjust the connection weights during supervised learning (learning by example) [4]. Learning occurs when the network weights are adjusted as a function of the error found in the output of the network. The error is the difference between the expected output and the actual output. The weights are adjusted backwards (backpropagated) through the ANN network until the error is minimized for a set of training data.

ANNs have been applied to an increasing number of realworld problems of considerable complexity. Their most important advantage is in solving problems that are too complex for conventional technologies; that is, problems that do not have an algorithmic solution or for which an algorithmic solution is too complex to be found. In general, because of their abstraction from the biological brain, ANNs are well suited to problems that people are good at solving, but for which computers are not. These problems include pattern recognition, modeling, and forecasting (which requires the recognition of trends in data).

The ANN approach to analysis of data will see extensive application to biophysical and biomedical problems in the next few years [5]. It has already been successfully applied to various areas of medicine, such as diagnostic aides, chemical analysis, image analysis, and drug development. The application of ANNs in diagnosing heart attacks received publicity when the ANN was able to make the diagnosis with a better accuracy than the physician [6]. This application is significant because it was used in the emergency room where the physician is not able to handle large amounts of data. A commercial product currently employs ANN technology to aid in the diagnosis of cervical cancer by examining pap smears [7]. In the United Kingdom, an ANN that is used in the early diagnosis of myocardial infarction is currently under going clinical testing at four hospitals [8], [9]. Other research level applications include diagnosis of heart murmur, diagnosis of coronary artery disease [10], ECG diagnosis [11], and detection of ischemic heart disease from exercise ECG tests [12].

\section{ANN Based Cardiovascular Modeling}

One approach to cardiovascular modeling is to build a model representative of a group of individuals with similar characteristics (i.e., sex, age, physical condition, medical condition, etc.). However, cardiovascular behavior is unique to each individual [13], thus a generic cardiovascular model used in a medical diagnostic system would not be as sensitive as a system based on a model that is adapted to the patient being diagnosed. To develop these models without a cardiovascular expert, the modeling must be based on an adaptive technology that can be automated. The ANN technology fits this category.

The ANN technology was selected for the cardiovascular modeling because of several capabilities including sensor fusion, which is the combining of values from several 
different sensors. Previous research has shown that ANNs have an inherit advantage over conventional analysis tools in processing the sensor information from multiple sensors. ANNs have the advantage that they process all the sensor values in parallel. This enables the ANNs to learn complex relationships among the individual sensor values, which would otherwise be lost if the values were individually analyzed. In medical modeling and diagnosis, this implies that even though each sensor in a set may be sensitive only to a specific physiological variable, ANNs are capable of detecting complex medical conditions by fusing the data from the individual biomedical sensors.

Another advantage of the parallel processing nature of ANNs is the speed. During development, ANNs are configured in a training mode, which is a repetitive process of presenting data from known diagnoses to a training algorithm. This training mode often takes many hours for large training sets. The payback occurs during the use of the model where propagation of the data through the system takes only a fraction of a second. Since this propagation time is similar to the response times of many sensors, this approach permits real-time diagnosis.

Recurrent ANNs were selected for this modeling to capture the temporal information in physiological variables [14]. These variables are time-series data from which both the absolute values and the rates of change need to be modeled. Recurrent ANNs have recurrent links or feedback lines that recycle a small portion of information from time $t-1$ at time $t$. Indirectly, decreasing portions of information from time $t-2, t-3, t-$ 4 , etc. are also captured by the model. This contextual information is what enables the recurrent ANNs to model the temporal dynamics of data. Figure 1 depicts the ANN configuration used in this research for modeling cardiovascular systems. Besides the general data flow through the ANN going directly from the input to the output, the figure shows that some lines bring information backwards in the ANN.

The ALS includes a training algorithm for the ANN. This algorithm receives physiological data from an individual via biomedical sensors during an adaptation phase. The algorithm automatically develops the model in the ANN during this phase. After the model is developed, it can generate the appropriate levels of the modeled physiological variables for simulations with varying levels of physical activity. Figure 2 illustrates the ALS as a modeling tool for physiological variables received from biomedical sensors attached to an individual. The ALS develops ANN based models from these variables.

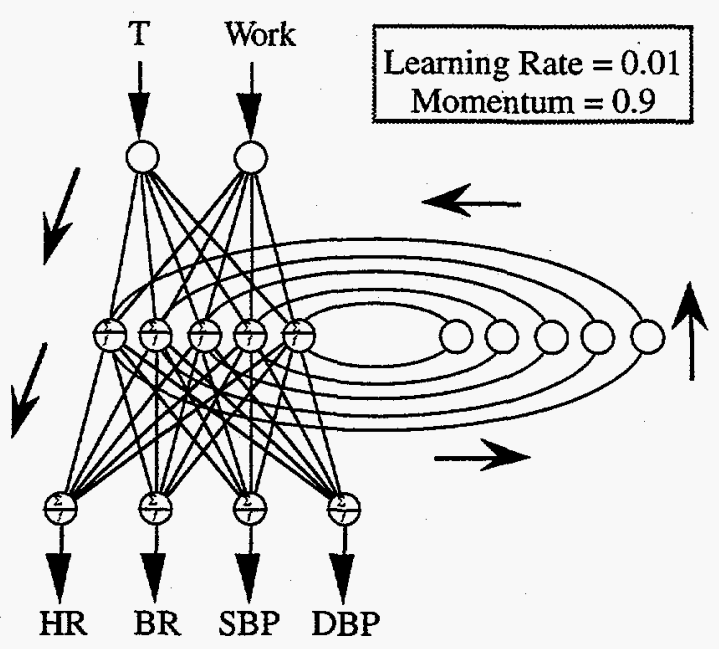

Figure 1. This figure illustrates the configuration of the ANN used in the cardiovascular modeling described in this paper. The ANN has two inputs, four outputs, and five hidden processing elements. The ANN takes the ambient temperature ( $\mathrm{T}$ ) and the physical activity (Work) as input. The four outputs, heart rate (HR), breathing rate (BR), systolic blood pressure (SBP), and diastolic blood pressure (DBP) are clamped to the "actual" values during the training phase. The "actual" values are generated by a nonadaptive cardiovascular model. During the modeling phase, the temperature and the work are input to the ANN, and the values at the outputs are taken as the modeled variables. The feedback links going through the five processing elements on the right side of the figure enables this ANN to capture the temporal information in the physiological variables.

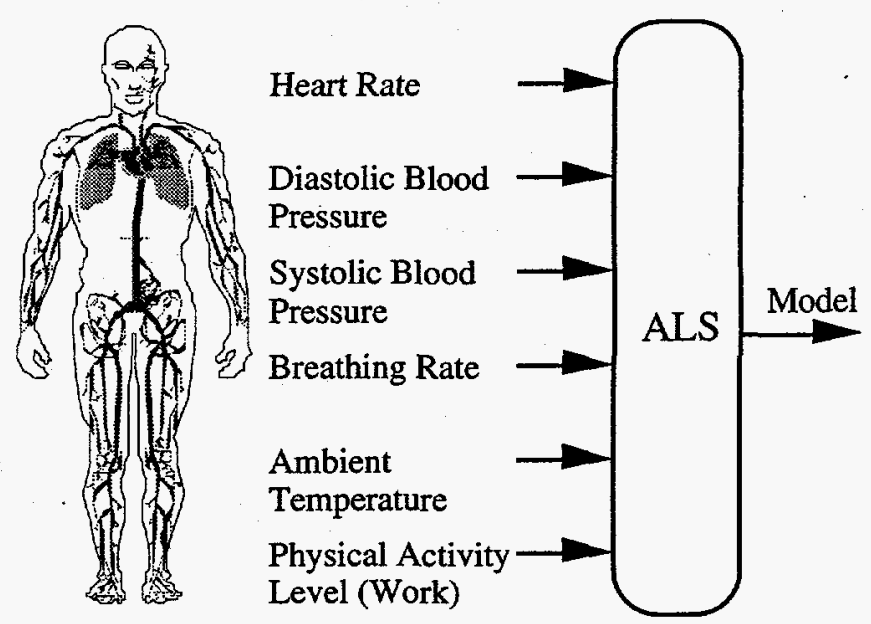

Figure 2. This figure illustrates the ALS as a tool for developing cardiovascular models. The modeling tool takes a sequence of physiological variables via biomedical sensors and learns the time varying dynamics of these variables.

Figure 3 shows how the variables modeled with the ANN compare with the physiological variables generated with a nonadaptive cardiovascular model [15], [16]. This second model has been used for creating data with sufficient complexity for the development of the ALS tool. 


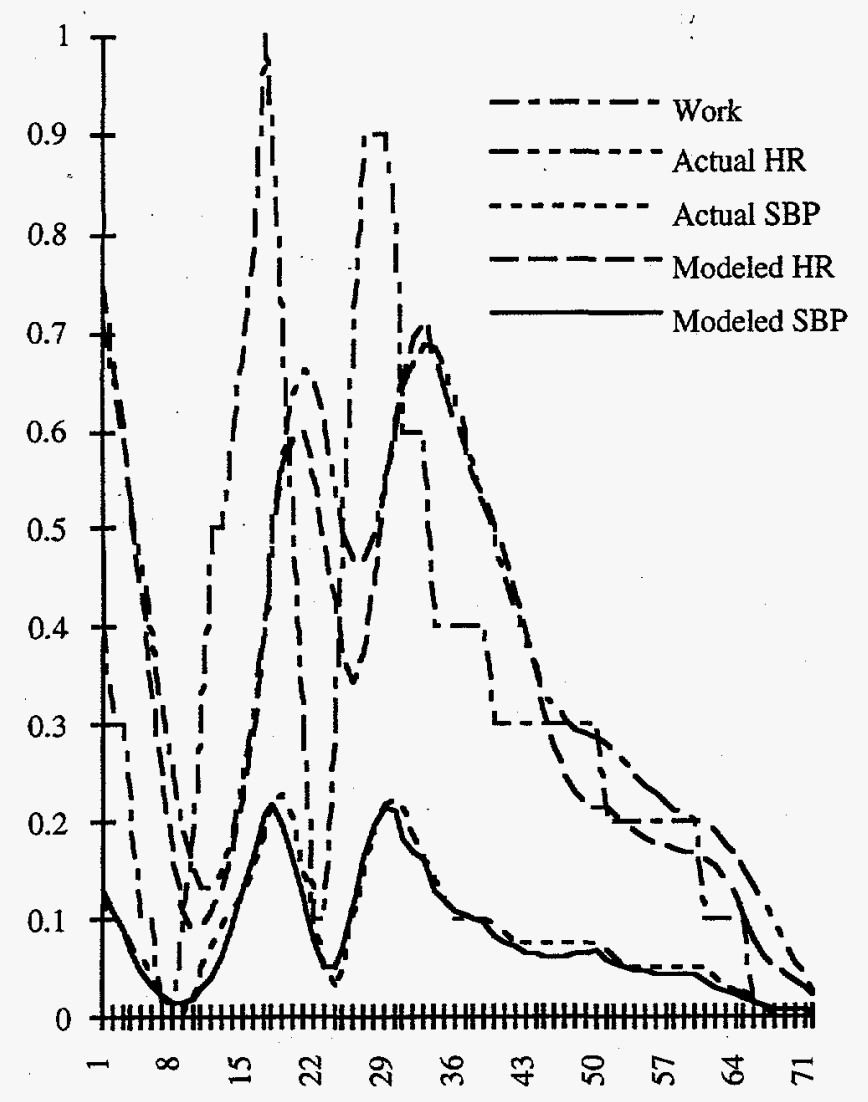

Figure 3. This graph depicts the "actual" and modeled heart rate (HR), and the "actual" and modeled systolic blood pressure (SBP) for varying physical activity levels (Work). The "actual" variables in this graph are generated with a nonadaptive cardiovascular model. The vertical axis corresponds to the normalized magnitude of these variables (normalized to one). The horizontal axis corresponds to the ordinal number of the sampled set of variables. The variables for systolic blood pressure and breathing rate are excluded from this figure for clarity. The effect of varying ambient temperature has not yet been explored in this research.

\section{Discussion}

In this paper, a prototype of ALS that models a subset of the cardiovascular system of an individual was presented. The ALS is a tool that models an individual's cardiovascular system as seen through the relationship between physiological variables and the level of physical activity. The modeling tool learns the dynamics of the relationship between these variables for an individual observed at different physical activity levels. Because the model adapts to an individual, it duplicates the physical condition of that individual. As such, it can be employed in "what-if" medical scenarios, to evaluate and diagnose of medical conditions and physical changes.

A modeling tool of this type is envisioned to serve in two broad areas. First, it would serve in a personal health diagnostic system for continuous diagnosis of health and for periodic clinical tests: graded exercise tests and stress tests of the cardiovascular system. For example, a real-time diagnostic system using these cardiovascular models may be used to monitor the health of workers in hazardous environments (e.g., law enforcement officers on duty and soldiers in battle) or to monitor and control administration of medication for hospital patients. Second, the modeling of biological systems are needed in, for example, education and research related to the human physiology and as a controller for medical mannequins.

In future work, this research will include the modeling of additional physiological variables, specifically variables describing pulmonary gas exchange: oxygen uptake $\left(\mathrm{V}_{\mathrm{O}_{2}}\right)$, and the concentrations of carbon dioxide $\left(\mathrm{CO}_{2}\right)$ and nitrogen $\left(\mathrm{N}_{2}\right)$. A complete physiological exercise test should also include multichannel electrocardiography (ECG).

Information on ANN developments at Pacific Northwest Laboratory is available in the World Wide Web (WWW) pages of the Environmental Molecular Science Laboratory which is accessible through such WWW clients as NCSA Mosaic.

http://www.emsl.pnl.gov:2080/docs/cie/neural/

\section{References}

[1] N. Jones, Clinical Exercise Testing, W.B. Saunders Company, Philadelpia, 3nd édition, 1988.

[2] D.R. Lamb, Physiology of Exercise: Responses and Adaptations, MacMillan Publishing Company, New York, 2nd edition, 1984.

[3] M.L. Pollock, J.H. Wilmore, Exercise in Health and Disease: Evaluation and Prescription for Prevention and Rehabilitation, W.B. Saunders Company, Philadelpia, 2nd edition, 1990.

[4] P. Werbos, "Beyond Regression: New Tools for Prediction and Analysis in the Behavioral Sciences," Ph.D. thesis, Harvard University, Cambridge, MA, 1974.

[5] D. Jones, "Neural Networks for Medical Diagnosis," Handbook of Neural Computing Applications, Academic Press, pp. 309-318, 1990.

[6] W.G. Baxt, "Use of an artificial neural network for data analysis in clinical decision-making: The diagnosis of acute coronary occlusion," Neural Computation 2, pp. 480-489, 1991.

[7] L.J. Mango, "Computer-assisted cervical cancer screening using neural networks," Cancer Letters, Vol. 77, No. 2 155-162, 1994.

[8] R.F. Harrison, S.J. Marshall, R.L. Kennedy, "The early diagnosis of heart attacks: a neurocomputational approach," Proceedings of the International Joint Conference on Neural Networks Intelligence (AIME), Maastricht, pp. 119-128, 1991. 
[9] R.L. Kennedy, Robert F. Harrison, S.J. Marshall, C.A., Hardisty, "Analysis of clinical and electrocardiographic data from patients with acute chest pain using a neurocomputer," Q J Med., Vol. 80, pp. 788789, 1991.

[10] C. Rosenberg, J. Erel, H. Atlan, "A Neural Network that Learns to Interpret Myocardial Planar Thallium Scintigrams," Neural Computation, Vol. 5, No. 3, 1993.

[11] Y. Suzuki, Y. Abe, K. Ono, "Self-organizing QRS Wave Recognition System in ECG using ART2," Proceedings of the World Congress on Neural Networks, Portland, OR, 11-15 July 1993, Vol. 4, pp. 39-42, 1993.

[12] G. Dorffner, E. Leitgeb, H. Koller, "Toward Improving Exercise ECG for Detecting Ischemic Heart Disease with Recurrent and Feedforward Neural Nets," Proceedings of the IEEE Workshop on Neural Networks in Signal Processing, Ermioni, Greece, 1994.

[13] A.J. Vander, J.H. Sherman, D.S. Luciano, Human Physiology: The Mechanisms of Body Function, McGraw-Hill Publishing Company, New York, 1990.

[14] B. Blumenfeld, "A Connectionist Approach to the Processing of Time Dependent Medical Parameters," Proceeding of the 1990 International Joint Conference on Neural Networks, Washington, DC, Vol. 2, pp. 575578, 1990.

[15] F.T. Tehrani, "Mathematical Analysis and Computer Simulation of the Respiratory System in the Newborn Infant," IEEE Transactions on Biomedical Engineering; Vol. 40, No. 5, pp. 475-481, 1993.

[16] A.Y.K. Wong and J.A. Armour, "A Neural Network Model of Canine Intrathoracic Ganglia Regulating the Heart," Neural Networks, Vol. 5, pp. 35-46, 1992. 